\title{
Optimización del tratamiento de fosfatado como método de protección frente a la corrosión ${ }^{(\bullet)}$
}

\author{
E. Onofre-Bustamante ${ }^{* * * *}$, A. Olvera*, A. Barba**, F.J. Rodríguez*, M. Carboneras ${ }^{* * *}$, M. Álvarez ${ }^{* * *}$, \\ M.C. García-Alonso ${ }^{* * *}$ y M.L. Escudero**
}

\begin{abstract}
Resumen
En el presente trabajo se estudia el fosfatado como tratamiento de conversión química (TCQ) libre de cromo, partiendo de un estudio básico del fosfatado comercial aplicado por inmersión en soluciones al $100 \%$ y diluciones 1:2, 1:5 y 1:10. Para conseguir un buen recubrimiento de fosfato se determinó el tiempo óptimo de inmersión mediante curvas de potencial a circuito abierto. Las propiedades de protección contra la corrosión se evaluaron mediante medidas de resistencia a la polarización e impedancia electroquímica. La adherencia al sustrato se determinó por ensayos de tracción (pull-off). Los resultados en inmersión continua mostraron que la aplicación de un recubrimiento orgánico sobre el sustrato previamente fosfatado en una dilución 1:5 (10 min) aumenta la resistencia a la corrosión en, al menos, un orden de magnitud con respecto al sustrato no fosfatado, incrementando la adherencia y el tiempo de vida del sistema recubrimiento-sustrato debido a que la película de conversión forma parte integral del sustrato y la superficie generada incrementa el área y número de sitios activos.
\end{abstract}

Palabras clave Fosfatado; Corrosión; Protección; Adhesión.

\section{Optimisation of the phosphate conversion treatment for generating protective coatings}

\begin{abstract}
This paper evaluates the phosphate treatment as a chemical conversion treatment (CCT) free of chromium, from the basis of the study of a commercial treatment applied in phosphoric solution at different concentrations $(100 \%$ and dilutions 1:2, 1:5 and 1:10). The optimum immersion time for the phosphate conversion treatment was established from the evolution over time of the open circuit potential. Protection against corrosion of the conversion layers was evaluated by polarisation resistance and electrochemical impedance measurements. The adhesion to the substrate was evaluated by pull-off tests. Results have shown that the application of an organic coating on the substrate with a previous phosphate treatment in 1:5 dilution (10 min.) increases the corrosion resistance in, at least, one order of magnitude with respect to the non phosphate-coated metal and improves the adhesion to the substrate, since the conversion layer obtained is part of the substrate and the surface generated increases the number and area of active sites.
\end{abstract}

Keywords

Phosphate; Corrosion; Protection; Adhesion.

\section{INTRODUCCIÓN}

En los últimos años, los tratamientos de conversión química (TCQ) han cobrado importancia como métodos de preparación de superficies, debido a los excelentes resultados obtenidos, principalmente, en sustratos como el aluminio ${ }^{[1-4]}$. Dentro de los TCQ comerciales más utilizados en la industria se encuentra el cromatado, el cual utiliza iones cromo en estado hexavalente, altamente tóxicos. La conciencia ecológica ha sido el principal detonante que ha motivado que parte de la comunidad científica dirija sus estudios a la búsqueda de nuevas alternativas, libres de cromo hexavalente. En este sentido, la

\footnotetext{
(•) Trabajo recibido el día 30 de Octubre de 2009 y aceptado en su forma final el día 2 de Marzo de 2010.

* Universidad Nacional Autónoma de México, Edificio D Facultad de Química Departamento de Metalurgia, Ciudad Universitaria, C. P. 04510 México D.F., México. e-mail: edonofre73@yahoo.com.mx.

** Universidad Nacional Autónoma de México, Edificio de Posgrado. Centro de Ingeniería de Superficies y Acabados (CENISA). Facultad de Ingeniería Departamento de Materiales y Manufactura, Ciudad Universitaria, C. P. 04510 México D.F., México.

*** Centro Nacional de Investigaciones Metalúrgicas, CENIM, Consejo Superior de Investigaciones Científicas, CSIC, Avda. Gregorio del Amo 8, 28040 Madrid, Spain
} 
introducción de nuevos TCQ que incluyen compuestos de cromo trivalente, fosfatos, tanatos, lantanos y tierras raras parecen ser las opciones vigentes que tienden a la conservación del medio ambiente $e^{[4-10]}$.

El fosfatado surgió como la primera alternativa al cromatado y es considerado una técnica antigua. De hecho, existen indicios de que los romanos del siglo III antes de nuestra era ya lo empleaban en la protección de ciertos artículos de hierro ${ }^{[11]}$. En 1869, se observó ${ }^{[12]}$ que la corrosión de las ballenas metálicas de los corsés se retrasaban tratándolas con ácido fosfórico. La primera aplicación importante de los recubrimientos de fosfato es consecuencia de las sugerencias de Thomas Watts Coslett ${ }^{[13-17]}$, quien, en 1908, empezó a tratar las piezas de hierro y de acero con una solución compuesta por ácido fosfórico y sulfato ferroso ${ }^{[13]}$. Más tarde, Coslett sustituyó el sulfato ferroso por el de zinc y, en 1918, la compañía Parker ${ }^{[18-21]}$ comprobó que el carácter del recubrimiento se mejoraba empleando una solución de sulfato de manganeso primario (parkerización), el dióxido de manganeso se añadía al baño, creyéndose que era esencial que, por lo menos, una parte del fosfato formase fosfato férrico. El tiempo que requiere el tratamiento oscila entre tres y cuatro horas. Recientemente y de acuerdo con la Directiva 004/73/CE de la Unión Europea, el fosfato de zinc, ha sido clasificado como peligroso para el medio, específicamente en efluentes acuosos ${ }^{[22]}$. Sin embargo, y a pesar del surgimiento de nuevas alternativas denominadas ecológicas o verdes, hoy en día, el fosfatado tiene una serie de aplicaciones importantes en muchas industrias, como la del automóvil, en donde es común que los segmentos de pistón, las levas, las válvulas, los tanques y las camisas de los cilindros se fosfaten para prolongar su vida en servicio. El tratamiento de fosfatado se emplea también en las pinturas y va desde su uso como pigmento inhibidor de la corrosión $^{[23}$ y 24], tratamientos de conversión quími$\mathrm{ca}^{[25-27]} \mathrm{y} / \mathrm{o}$ pre-tratamiento ${ }^{[22,28}$ y 29], que funciona como base para las pinturas y aumenta su adherencia al sustrato.

Por todo esto, el fosfatado se sigue considerado la primera alternativa al cromatado que no presenta problemas severos de toxicidad, pero que no proporciona las mismas propiedades anticorrosivas que el cromatado ${ }^{[9-11}$ y $\left.30-32\right]$ debido, principalmente, al díficil control de las variables involucradas en el proceso (concentración, temperatura y $\mathrm{pH}$ ).

Por tanto, el fosfatado como TCQ representa, en la actualidad, una alternativa para solucionar o mejorar problemas medioambientales, planteando nuevos retos en distintos campos como el de los TCQ y los pigmentos inhibidores, sin embargo, es indiscutiblemente necesario un estudio y control de las variables involucradas en el proceso de fosfatado. En este sentido, el objetivo del presente trabajo consiste en estudiar y optimizar el tratamiento de fosfatado como TCQ partiendo de un estudio básico del fosfatado comercial, aplicado por inmersión en soluciones al $100 \%$ y en diluciones 1:2, 1:5 y 1:10 a partir de una solución comercial ELECTROCHEM ${ }(\mathrm{pH}=1)$ a temperatura ambiente. El estudio ha consistido en la determinación de la concentración y tiempo óptimo de inmersión en las distintas soluciones de fosfatado y en la evaluación de la resistencia frente a la corrosión generada por las películas de conversión obtenidas. Asimismo se ha realizado una caracterización física y química de la superficie modificada para, finalmente, determinar la resistencia a la corrosión de dichos recubrimientos en un medio clorurado y la adherencia generada por los TCQ en la intercara recubrimiento-sustrato mediante ensayos de tracción (pull-off).

\section{CONDICIONES EXPERIMENTALES}

\subsection{Preparación y tratamiento superficial de las muestras}

Todos los experimentos fueron realizados sobre placas de acero comercial AISI 1010, cuya composición elemental en \% en peso es: Fe-99.54, C-0.0840, Si0.0321, Mn-0.2371, P-0.0103, Al-0.0411 y Mo0.0372 . Antes de que las muestras fueran tratadas, se desengrasaron con detergente y agua destilada. Para eliminar restos de agua, las muestras fueron secadas con acetona.

Los tratamientos se aplicaron por inmersión utilizando la solución fosfatante comercial ELECTRO$\mathrm{CHEM}^{\circledR}$ al $100 \%$, con un $\mathrm{pH}=1$ y una densidad de $1,23 \mathrm{~g} / \mathrm{ml}$, compuesta, principalmente, por ácido fosfórico y sulfato de manganeso, este último en una concentración máxima de 10 a 20 g/l. Esta solución esta especificada por el proveedor como desoxi-fosfatante, lo que implica que es una solución capaz de realizar una limpieza superficial (decapado) y, posteriormente, depositar una película de fosfato que favorece la adherencia de recubrimientos posteriores y es utilizada de manera industrial, principalmente sobre sustratos ferrosos. El proveedor indica que el tratamiento óptimo en una solución al $100 \%$ se obtiene por inmersión durante $30 \mathrm{~min}$ a $50^{\circ} \mathrm{C}$, consiguiéndose resultados similares en menor tiempo incrementando la temperatura hasta $70^{\circ} \mathrm{C}$. A partir de la solución al $100 \%$ se prepararon diluciones $1: 2,1: 5$ y $1: 10$, utilizando agua destilada grado analítico a temperatura 
ambiente para determinar la concentración óptima de fosfatado.

\subsection{Caracterización superficial}

La morfología y composición química de la película de conversión generada, antes de aplicar el recubrimiento final de tipo orgánico, fue estudiada mediante microscopía electrónica de barrido (SEM), utilizando un equipo JEOL JSM-35C equipado con EDX Voyager Tracor Northern Spectrometer. Asimismo, se realizaron medidas de difracción de rayos X (XRD), empleando un difractómetro Siemens 5000, operando a $35 \mathrm{kV}$ y $25 \mathrm{~mA}$ con un monocromador cristalino de grafito, utilizando una lámpara de cobre. Las muestras fueron exploradas a una velocidad de $2^{\circ}$ $\min ^{-1}$ entre 20 y $100^{\circ}$.

\subsection{Ensayos electroquímicos}

Para determinar el tiempo óptimo de inmersión en la solución fosfatante se realizaron ensayos de registro del potencial a circuito abierto (OCP) para las placas de acero al carbono, empleando como electrolito las distintas soluciones evaluadas (solución al $100 \%$ y diluciones $1: 2,1: 5$ y $1: 10$ ). Estas medidas electroquímicas se realizaron mediante un potenciostato-galvanostato VIMAR modelo 352, tomando lecturas del potencial cada $30 \mathrm{~s}$ hasta que el potencial de corrosión presentaba un valor estable.

La resistencia a la corrosión de las muestras tratadas se evaluó mediante medidas de resistencia a la polarización $\left(\mathrm{R}_{\mathrm{p}}\right)$ y de espectroscopía de impedancia electroquímica (EIS), utilizando un equipo Gill-AC modelo 802 acoplado a una computadora. Las medidas de $R_{p}$ fueron determinadas de acuerdo con la norma ASTM G-59-97, aplicando una polarización de $\pm 20 \mathrm{mV}$, con respecto al potencial de corrosión, con una velocidad de barrido de $0,1 \mathrm{mV} / \mathrm{s}$. Las medidas de EIS se llevaron a cabo aplicando una señal sinusoidal de $10 \mathrm{mV}$ de amplitud en un barrido de frecuencia de 103 hasta $10^{-3} \mathrm{~Hz}$, tomando 10 puntos por década de frecuencia. En ambas técnicas, se utilizó como electrolito una solución de $\mathrm{NaCl}$ al $3 \%$ en peso, simulando un ambiente sumamente agresivo, preparada a partir de $\mathrm{NaCl}$ de grado analítico y agua destilada. Se trabajó con una celda convencional de tres electrodos, utilizando un electrodo de calomelanos saturado $(0,2415 \mathrm{~V}$ vs SHE) como electrodo de referencia y una barra de grafito como contraelectrodo. El área del electrodo de trabajo para los ensayos de Rp y EIS fue 1.23 y $8.5 \mathrm{~cm}^{2}$, respectivamente.

\subsection{Ensayos de adherencia}

Para poder discernir el efecto de los tratamientos de conversión química sobre la adherencia del sistema metal-pintura, las placas de acero fueron pintadas con un barniz transparente de base poliuretano, utilizando una cuchilla BYK Gardner Serie AG-4300 para tener un control del espesor de pintura. Se aplicaron espesores de película húmeda de 100 micras para obtener finalmente un espesor de 40 a $50 \mathrm{mi}$ cras de película seca, tomando como referencia que el recubrimiento que se utilizó posee un 44-46 \% de sólidos en peso. Un medidor de espesores marca ELCOMETER modelo 345 fue utilizado para cuantificar el espesor de película seca.

La adherencia del sistema metal + capa de fosfato + recubrimiento orgánico fue evaluada mediante ensayos de tracción, método pull-off, según la norma ASTM D-4541. Placas de acero desnudo, sin tratamiento de fosfatado, también fueron pintadas y evaluadas bajo las mismas condiciones.

\section{RESULTADOS Y DISCUSIÓN}

\subsection{Cinética de crecimiento de la capa de fosfato mediante técnicas electroquímicas}

La figura 1 muestra la evolución del potencial de corrosión $\left(\mathrm{E}_{\text {corr }}\right)$ y la resistencia a la polarización $\left(\mathrm{R}_{\mathrm{p}}\right)$ en función del tiempo de inmersión, respectivamente, para muestras de acero al carbono evaluadas en las diferentes soluciones fosfatantes. En la figura 1 a) se puede observar que el valor de Ecorr en todas las muestras evaluadas es muy estable, permaneciendo prácticamente constante después de $8 \mathrm{~min}$ (valores activos comprendidos entre -480 y $-570 \mathrm{mV}$ ); este comportamiento del potencial de corrosión, relativamente estable, parece correlacionarse con la finalización de la formación de la película de fosfato, $\mathrm{N}$. Btretherton et al. ${ }^{[33]}$ encontraron un comportamiento similar al estudiar la formación de películas de fosfato sobre sustrato de zinc y sugieren el uso de agentes aceleradores, como nitritos u oxidantes fuertes para reducir el tiempo necesario para la formación de película de fosfato.

Con respecto a la solución al 100 \%, la muestra evaluada en la solución fosfatante 1:2 es la única que presenta valores de potencial más anódicos. La evolución del potencial de la muestra evaluada en la disolución 1:5 presenta un comportamiento muy similar al observado para la muestra en la solución al $100 \%$. Según se observa en la figura 1 b), los 

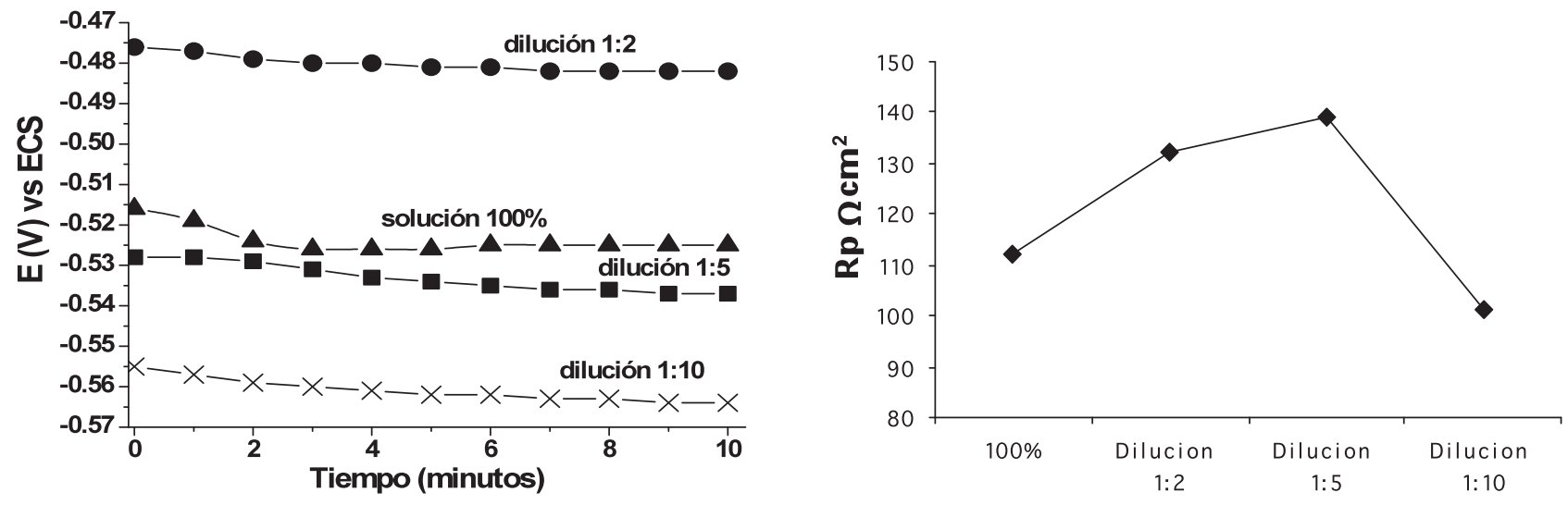

Figura 1. Variación del potencial en función del tiempo (a) y valores de resistencia a la polarización (b) del acero tratado en diferentes soluciones fosfatantes.

Figure 1. Evolution of potential over time (a) and polarisation resistance values (b) for the steel treated in different phosphate solutions.

valores de Rp obtenidos se encuentran comprendidos entre 100 y $150 \Omega \mathrm{cm}^{2}$, sin presentarse variaciones significativas concluyentes entre los diferentes tratamientos aplicados. Comparativamente, los valores de $R_{p}$ indican que el medio más agresivo es el más diluido (1:10), resultado también observado a partir de las medidas de $\mathrm{E}_{\text {corr }}$.

Los valores bajos de $\mathrm{Rp}$ obtenidos se deben a la naturaleza ácida de la solución fosfatante (ácido fosfórico), con valor de $\mathrm{pH}$ entre 0,8 y 1 , que ataca al sustrato metálico rápidamente, lo que sin duda justifica que en todos los baños utilizados se presente una cinética de formación de la película muy elevada. Sin embargo, es importante resaltar que las muestras tratadas en la solución fosfatante 1:2 presentan un valor de potencial más anódico y el valor de Rp no es muy distinto del obtenido en la solución 1:5. Este hecho podría ser contradictorio, sin embargo, la diferencia de espesores de la película de fosfato obtenida en la solución 1:5 podría ser la causa de un incremento en el valor de $\mathrm{R}_{\mathrm{p}}$.

En conclusión, de los resultados obtenidos (Fig. 1 a)) se puede determinar que el tiempo óptimo de inmersión en cualquiera de las soluciones fosfatantes es de $10 \mathrm{~min}$, a partir del cual se alcanza un valor de potencial de corrosión estable o, lo que es lo mismo, un estado estacionario del sistema en estudio y, por tanto, se asegura que la formación de la película de fosfato ha concluido. Las películas de conversión obtenidas en la disolución 1:5 podrían presentar un grado de protección similar a las obtenidas en la solución al $100 \%$, un resultado que, de ser corroborado, permitiría una optimización del proceso, disminuyendo el tiempo de tratamiento y concentración que el proveedor proponía, lo que se reflejaría en un menor coste económico.

\subsection{Caracterización morfológica de las películas de fosfato}

Las películas de conversión obtenidas fueron evaluadas mediante difracción de rayos $\mathrm{X}$ y se determinó que están formadas por fosfato de hierro secundario (Fig. 2) y una pequeña cantidad de magnetita. La magnetita presente en las películas de conversión podría contribuir a la adherencia del recubrimiento al sustrato, debido a que es muy porosa y adherente, como ha sido establecido en la literatura ${ }^{[30}$ y 34]. Puesto que las películas de conversión obtenidas son muy delgadas, los picos que se relacionan con la formación del fosfato de hierro presentan una baja intensidad; sin embargo, coinciden con lo publicado en la literatura ${ }^{[31]}$.

El estudio mediante SEM corroboró los resultados obtenidos por EDX, los cuales detectaron la presencia de una película de fosfato de hierro homogénea con diminutas zonas ricas en fósforo (Fig. 3 a)). Para poder determinar los espesores del fosfatado mediante SEM se provocó un defecto localizado sobre la película de conversión, en todas las muestras (Fig. 3 b)), empleando una broca de $50 \mu \mathrm{m}$ de diámetro y se realizaron análisis EDX en las zonas interior y exterior del defecto. En el interior del defecto, solo se detectó oxígeno y hierro, no siendo detectada la presencia de fósforo, resultado que confirma que la zona analizada corresponde al sustrato de acero. En la zona exterior del defecto, cerca del borde, los análisis detectaron mayores cantidades de 


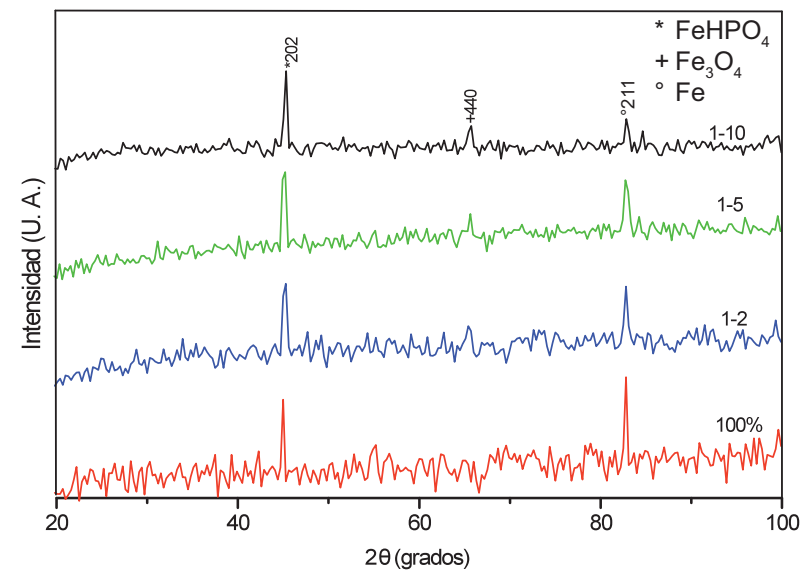

Figura 2. Difractograma de las muestras de acero fosfatadas en diferentes soluciones (solución al $100 \%$ y diluciones $1: 2,1: 5$ y $1: 10$ ).

Figure 2. XRD spectrum for the steel samples after the phosphate treatment in different solutions (solution 100\% and dilutions 1:2, 1:5, and 1:10).

oxígeno y fósforo, así como trazas de hierro, corroborando que la zona analizada corresponde a la película de conversión. Posteriormente, los espesores de película se determinaron tomando como base la altura en el borde del defecto (Fig. 3 c)), aunque solo para la muestra tratada en la solución fosfatante 1:5 se pudo determinar el espesor, ya que las demás muestras presentaban películas demasiado delgadas, con espesores por debajo del límite de detección del equipo utilizado. Como se muestra en la figura $3 \mathrm{c}$ ), los espesores de película registrados para las muestras tratadas en la solución fosfatante 1:5 se encuentran comprendidos entre 1 y 2 micras.

Por otro lado, partiendo de que el baño de conversión está constituido principalmente por ácido fosfórico y una pequeña cantidad de fosfato de zinc, el proceso o mecanismo de formación de la película de conversión se basa en la reacción del ácido fosfórico libre en solución con el hierro, para formar fosfato de hierro secundario y liberar hidrógeno en las zonas catódicas de acuerdo con las siguientes reacciones:

$$
\begin{gathered}
\mathrm{Fe}^{\mathrm{O}}+2 \mathrm{H}_{3} \mathrm{PO}_{4} \rightarrow \mathrm{Fe}\left(\mathrm{H}_{2} \mathrm{PO}_{4}\right)_{2}+2 \mathrm{H}++2 \mathrm{e}^{-} \\
2 \mathrm{H}^{+}+2 \mathrm{e}^{-} \rightarrow \mathrm{H}_{2} \\
\mathrm{Fe}\left(\mathrm{H}_{2} \mathrm{PO}_{4}\right)_{2} \rightarrow \mathrm{FeHPO}_{4}+\mathrm{H}_{3} \mathrm{PO}_{4}
\end{gathered}
$$

dando como resultado la formación de una película constituida, principalmente, por fosfato de hierro secundario, porosa y que otorga una buena cobertura al sustrato. La composición de la solución es crítica, pues si existe demasiado ácido libre la superficie de acero sólo se decapa, mientras que si dicha cantidad es pequeña, se fomenta la formación de lodos ${ }^{[35 \text { y } 36]}$. No obstante, el desprendimiento de hidrógeno durante el fosfatado puede impedir el acceso del acido a la interfase, ralentizando la formación de la capa pasiva, siendo necesario la utilización de un agente oxidante que reaccione con el hidrógeno para formar agua ${ }^{[35]}$. En este caso, el acelerador podría no estar presente en la composición química de la solución fosfatante. Esto último podría ser la razón por la cual se obtienen películas delgadas. Apoyando

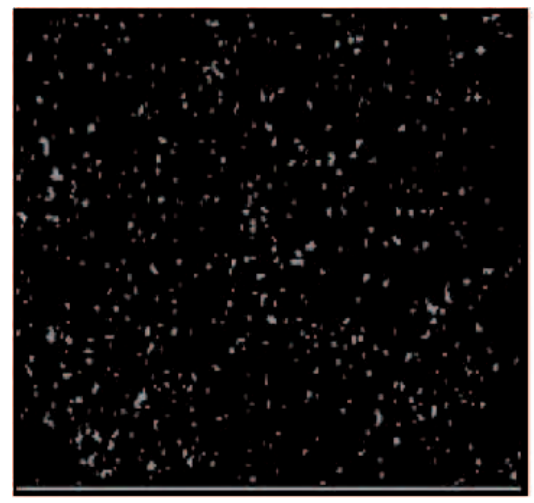

(a)

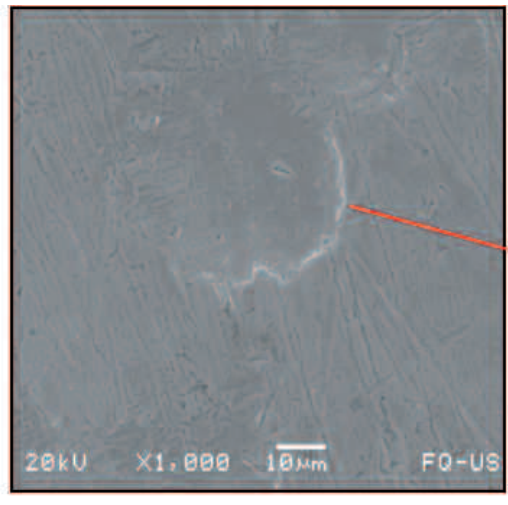

(b)

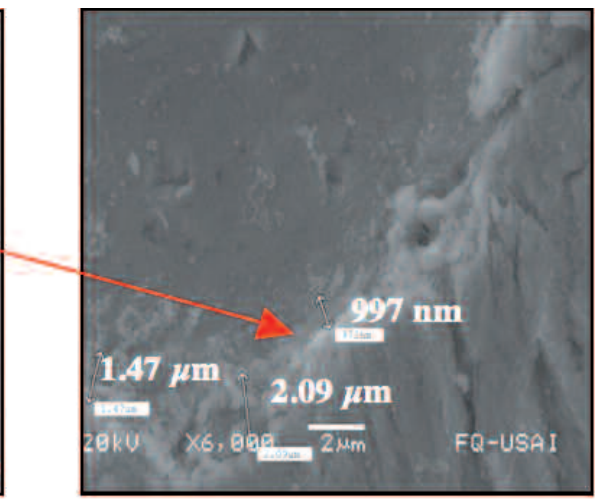

(c)

Figura 3. Imagen SEM para la muestra de acero tratada durante 10 min en solución fosfatante 1:5. (a) Mapeo de fósforo, (b) Defecto sobre la película de conversión y (c).Espesores de película.

Figure 3. SEM image for the steel sample after the phosphate treatment in 1:5 solutions for 10 min. (a) Phosphorous mapping, (b) Defect on the conversion coating and (c) Thickness of the layer. 
esta hipótesis, al disminuir la concentración de la solución fosfatante original, la cantidad de hidrógeno desprendida también disminuye, favoreciendo el crecimiento de la película de conversión, hecho que podría explicar porque las placas tratadas en una solución 1:5 son las que presentan mayor espesor de película de conversión; sin embargo, por debajo de esta concentración, si bien disminuye aun más la evolución de hidrógeno que ralentiza el crecimiento de la capa, la concentración de iones fosfato presentes en la disolución podría ser no suficiente para formar la película de conversión, requiriendo de mayor tiempo para el crecimiento de la capa.

A pesar de este inconveniente, las películas de conversión obtenidas presentan, como principal ventaja, que durante el proceso de formación una parte del hierro se convierte en parte integral del recubrimiento $^{[35 \text { y 36] }}$, formando fosfato de hierro, proporcionándole una excelente adherencia de tipo químico en la intercara película de conversión-sustrato. Este tipo de adherencia ha sido mostrada como la de mayor fuerza ${ }^{[37]}$, sin menospreciar la resistencia a la corrosión adicional que dicho tratamiento le confiere al acero, lo que podría corroborarse con los resultados electroquímicos obtenidos y los de adherencia que más adelante se discuten.

\subsection{Caracterización electroquímica de las películas de fosfato}

Una vez aplicado el tratamiento de fosfatado durante $10 \mathrm{~min}$, el comportamiento frente a la corrosión de las muestras de acero al carbono fue evaluado en

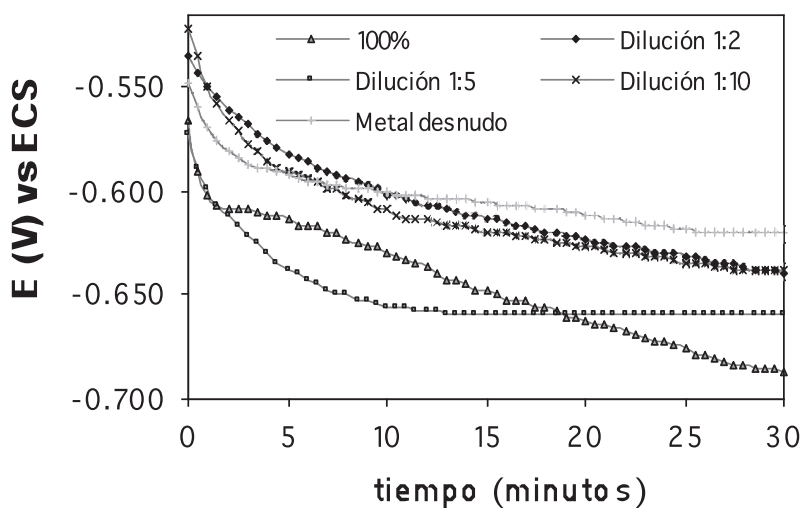

una solución de $\mathrm{NaCl}$ al $3 \%$ en peso. La figura 4 muestra los valores de $\mathrm{E}_{\text {corr }}$ y $\mathrm{R}_{\mathrm{p}}$ para las muestras tratadas en las diferentes soluciones fosfatantes con respecto al material desnudo, evaluado en igualdad de condiciones. Se puede observar que todas las películas obtenidas ofrecen cierto grado de protección, aunque el valor máximo se alcanza en la solución 1:5. Según muestra la figura 4 a), la película de conversión obtenida en la solución fosfatante 1:5 presenta valores de potencial de corrosión muy estables después de 10 min, estabilización que va acompañada de los valores más altos de $\mathrm{Rp}$ (Fig. 4 b)).

A diferencia del seguimiento del potencial de corrosión en los baños de conversión, los estudios de velocidad de corrosión después de que la película de fosfato se ha formado, proporcionan una medida de la penetración del electrólito a través de los recubrimientos de fosfato. N. Bretherton et al., en conjun-

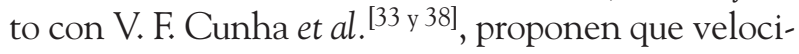
dades de corrosión elevadas están relacionadas en gran medida con una alta porosidad en la película o una significativa disolución de la película de fosfato. Apoyándose en esto, se podría decir que las películas más porosas son las que presentan menor $\mathrm{R}_{\mathrm{p}}$ (disolución $100 \%$ y 1:10) mientras que las películas obtenidas en la solución fosfatante 1:2 y 1:5 serían las menos porosas o con mejor cobertura del sustrato.

Comparativamente, las muestras de acero desnudas muestran valores de $680 \Omega \mathrm{cm}^{2}$ frente a las tratadas en la solución fosfatante 1:5, donde los valores de $\mathrm{Rp}$ son superiores a $1.200 \Omega \mathrm{cm}^{2}$.

El estudio de dichas muestras mediante EIS se muestra en la figura 5, en la que se presenta el diagrama de impedancia de Bode para las muestras desnudas y con los distintos tratamientos de fosfatado. En general,

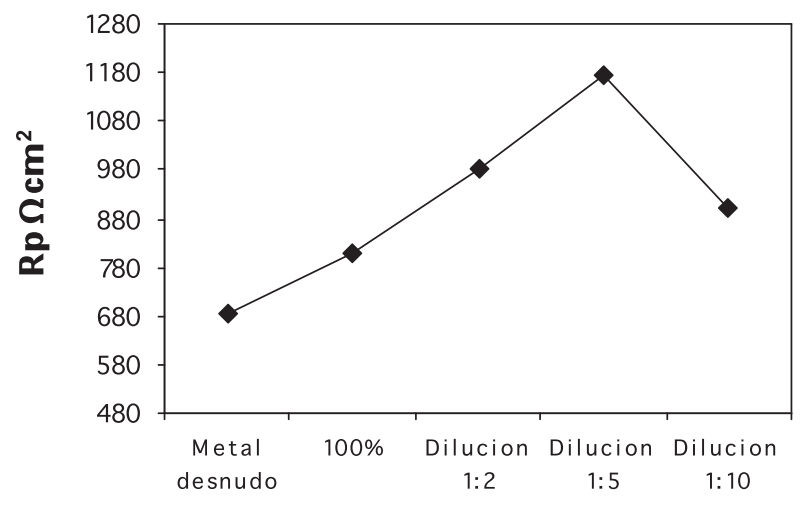

Figura 4. Valores de potencial en función del tiempo (a) y de resistencia a la polarización (b) obtenidos en una solución de $\mathrm{NaCl}$ al $3 \%$ en peso para las muestras de acero fosfatadas.

Figure 4. Potential values as a function of time (a) and polarization resistance values (b) in $3 \%$ wt $\mathrm{NaCl}$ solution for the steel samples after the phosphate treatments. 

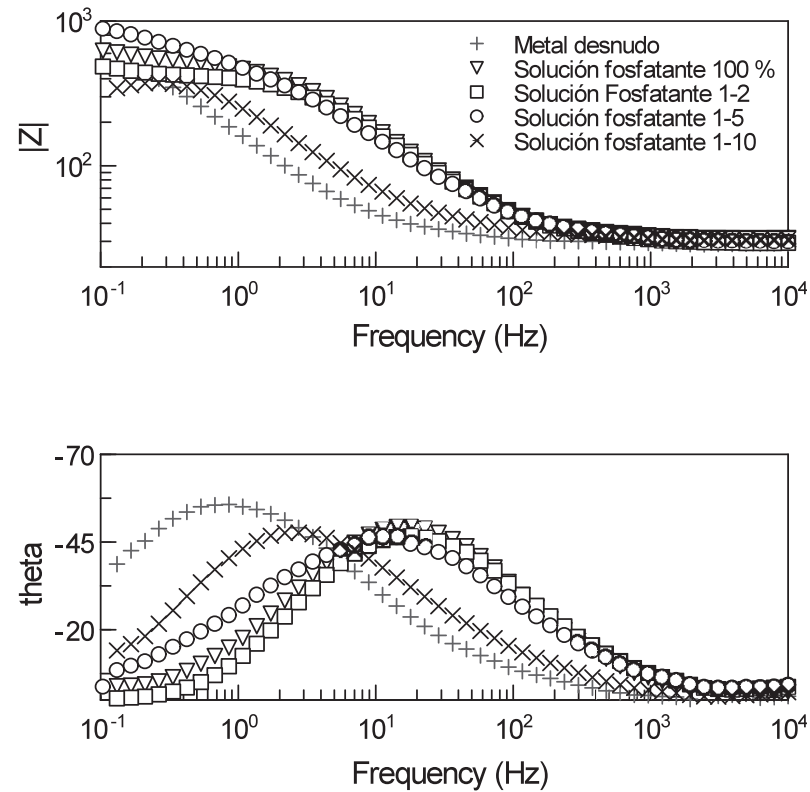

Figura 5. Diagramas de impedancia de Bode obtenido en una solución de $\mathrm{NaCl}$ al $3 \%$ en peso para las muestras de acero fosfatado.

Figure 5. Impedance diagram of Bode in $3 \%$ wt $\mathrm{NaCl}$ solution for the steel samples after the phosphate treatments.

los resultados presentan una sola pendiente en el diagrama de Bode, mostrando un módulo de impedancia en bajas frecuencias que oscila entre $10^{2}$ y $10^{3} \Omega \mathrm{cm}^{2}$. Dichos resultados corroboran los previamente obtenidos de las medidas de $R_{p}$ ya que, si bien no son exactamente los mismos valores, presentan la misma tendencia. De igual forma, se puede apreciar que el módulo de impedancia conseguido en la disolución 1:5 muestra un valor aproximado de $10^{3} \Omega \mathrm{cm}^{2}$, siendo este valor el más alto registrado con respecto a las demás concentraciones estudiadas.

Los resultados electroquímicos obtenidos se pueden explicar considerando, por un lado, que durante el fosfatado, como fue explicado anteriormente, al disminuir la concentración de la solución fosfatante original, la cantidad de hidrógeno desprendida disminuye, favoreciendo el crecimiento de la película de conversión. Este hecho afirma por qué las placas tratadas en una solución 1:5 son las que presentan mayor espesor, lo que le confiere una mayor resistencia a la corrosión, debido principalmente a un efecto barrera; sin embargo, por debajo de esta concentración, los iones fosfato disminuyen y la película de conversión es más delgada y se reduce su resis-

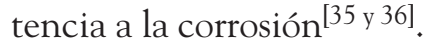

Por otro lado, la película de conversión puede ser vista como una red de $\operatorname{poros}^{[35 \text { y } 37]}$ distribuidos de manera uniforme e interconectados de modo que, dependiendo principalmente de su tamaño, será más fácil que el electrólito o los iones agresivos penetren a través de esta red, lleguen al sustrato y se lleve a cabo el

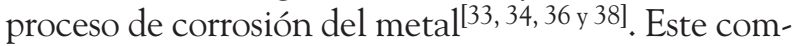
portamiento debería tener una respuesta de impedancia característica, separando la contribución de la capa de fosfato o película de conversión (dos constantes de tiempo). Sin embargo, debido a que la película es demasiado porosa, el electrolito es capaz de alcanzar inmediatamente la superficie del sustrato, produciéndose la reacción de corrosión. Por esta razón, aparece una sola pendiente en el diagrama de Bode, lo que indica que la constante de tiempo del fosfato es del mismo orden que la de reacción de corrosión y, por tanto, lo que se observa en este diagrama es la suma de ambas contribuciones y/o constantes de tiempo (película de conversión y reacción de corrosión).

\subsection{Ensayos de adherencia (pull-off)}

Los ensayos de adherencia se llevaron a cabo en muestras de acero, tratadas durante $10 \mathrm{~min}$ en las soluciones fosfatantes y, posteriormente, pintadas. Los resultados de la prueba de adherencia muestran que las placas tratadas en la solución fosfatante 1:5 requieren de una fuerza promedio de 9,7 MPa para desprender el $100 \%$ del área evaluada, mientras que para las muestras restantes (solución al 100 \% y diluciones $1: 2$ y $1: 10$ ) la fuerza requerida es menor, del orden de 2,3 $\mathrm{MPa}$. Por otro lado, las muestras de acero desnudo sin tratamiento de fosfatado presentaron valores de adherencia de 1,9 a 2,1 $\mathrm{MPa}$, muy inferiores a los publicados para la placa tratada en la solución fosfatante 1:5. Esto indica, nuevamente, que la unión del recubrimiento al sustrato originada por la capa de fosfato es considerablemente mayor. Estos excelentes valores son el resultado de la adherencia química presente en la intercara película de fosfato-sustrato y de la adherencia mecánica en la intercara película de fosfato-recubrimiento. Debido a la rugosidad y/o perfil de anclaje obtenido mediante el fosfatado, se produce una excelente integración del sistema sustrato metálico + capa de fosfato + recubrimiento orgánico, lo que se ve reflejado en un tiempo de vida mayor.

\subsection{Caracterización del sistema recubrimiento-sustrato en inmersión continua}

Muestras fosfatadas y pintadas con un barniz transparente se evaluaron en inmersión continua durante dos semanas, utilizando como electrolito $\mathrm{NaCl}$ al 


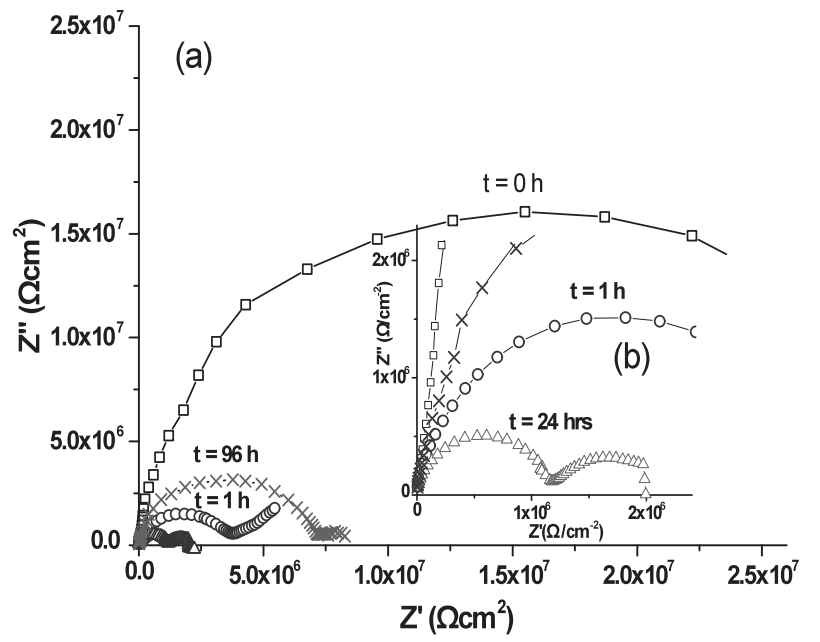

Figura 6. (a) Diagrama de Nyquist obtenido en solución de $\mathrm{NaCl}$ al $3 \%$ en peso para el acero pintado sin tratamiento previo de fosfatado, (b) Ampliación de la Figura (a).

Figure 6. Nyquist diagram in $3 \%$ wt $\mathrm{NaCl}$ solution for the painted steel sample with no previous phosphate treatment, (b) Zoom of figure (a).

$3 \%$ en peso, para determinar el efecto del fosfatado sobre la resistencia a la corrosión del sistema recubrimiento-sustrato. Con fines comparativos, muestras de acero sin fosfatar previamente a la aplicación del barniz, también fueron evaluadas en igualdad de condiciones.

En el diagrama de Nyquist (Fig. 6) se puede observar que los valores de impedancia del sistema sustrato desnudo + barniz disminuyen, significativamente, de $3 E 7 \Omega \mathrm{cm}^{2}$ a 3,8E6 $\Omega \mathrm{cm}^{2}$, en la primera hora de ensayo. También se puede apreciar que la impedancia sigue disminuyendo hasta un valor de $1,2 \mathrm{E} 6 \Omega \mathrm{cm}^{2}$ y que empieza a formarse una segunda constante de tiempo (semicírculo) a bajas frecuencias, para el primer día de evaluación, lo que es evidencia de que el electrolito atravesó el recubrimiento (barniz transparente) y, ahora, se encuentra en contacto directo con el sustrato metálico. A partir del segundo día de ensayo se aprecia un incremento en la impedancia del sistema en evaluación, aproximadamente $7 \mathrm{E} 6 \Omega \mathrm{cm}^{2}$. Este comportamiento se puede atribuir a la formación de productos de corrosión en la intercara recubrimiento-sustrato que podrían estar sellando los poros del recubrimiento permitiendo, así, que estos productos de corrosión junto con el recubrimiento actúen como una barrera física para el electrolito ${ }^{[34}$ y 39$]$. Sin embargo, esta barrera será vencida y la consecuencia final se presentará como un daño, ya sea por ampollamiento y/o delaminación del recubrimiento (barniz). No obstante, es importante

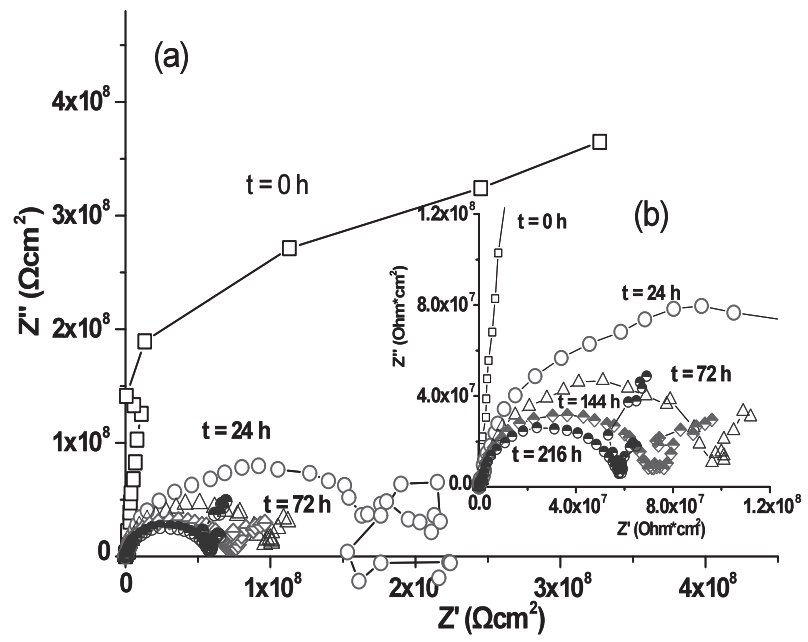

Figura 7. (a) Diagrama de Nyquist obtenido en solución de $\mathrm{NaCl}$ al $3 \%$ en peso para el acero pintado tras recibir tratamiento de fosfatado (10 min en solución 1:5), (b) Ampliación de la Figura (a).

Figure 7. Nyquist diagram in $3 \%$ wt $\mathrm{NaCl}$ solution for the painted steel sample with previous phosphate treatment (10 min in 1:5 solution), (b) Zoom of figure (a).

comentar que la calidad del recubrimiento no es buena aunque, para los fines de este trabajo, este comportamiento facilita la interpretación de los resultados ya que el interés principal del estudio se centra en evaluar la capacidad del tratamiento de conversión química (fosfatado), como enlace entre el sustrato y el recubrimiento final y su efecto en la resistencia a la corrosión y la adherencia de dicho sistema.

Los resultados correspondientes al sistema formado por el sustrato fosfatado en la solución 1:5 + barniz (Fig. 7) muestran un incremento de, al menos, un orden de magnitud $\left(1,8 \mathrm{E} 8 \Omega \mathrm{cm}^{2}\right)$, con respecto al acero desnudo. Este incremento solo se puede atribuir a la presencia del TCQ (fosfatado), ya que los dos sistemas de estudio fueron realizados en las mismas condiciones. Con el aumento del tiempo de ensayo los valores de impedancia comienzan a descender hasta alcanzar el valor de $5,8 \mathrm{E} 7 \Omega \mathrm{cm}^{2}$, mostrando, sólo, un semicírculo. A partir del noveno día se puede apreciar en el diagrama de Nyquist la presencia de un segundo semicírculo o constante de tiempo, que no alcanza a definirse bien y que podría asociarse a la película de conversión. En este caso, el tiempo de vida del sistema sustrato fosfatado + recubrimiento aumenta, presentando un menor daño en $9 \mathrm{~d}$ que el reportado para el caso del acero desnudo, que se degradó en las primeras 24 h de evaluación.

El incremento de la resistencia a la corrosión y el tiempo de vida del sistema metal + capa de fosfato 
+ recubrimiento orgánico se debe, principalmente, a que el TCQ crea sobre el acero una película rugosa con una microporosidad homogénea, que opera como un perfil de anclaje al aplicar el recubrimiento final (barniz), permitiendo que éste sea absorbido a través de los poros presentes en dicho perfil, ocupe los espacios generados por el mismo y ancle el recubrimiento, promoviendo una mayor adherencia mecánica en la intercara recubrimiento-fosfato y adherencia química entre el sustrato y la capa de fosfatado, proyectando una adherencia total mayor que la obtenida para el acero desnudo sin fosfatar (Tabla II). Conjuntamente con esto, el fosfatado se consolida con el barniz para formar una barrera mecánica más estable, compuesta por dos capas (TCQ y barniz) perfectamente adheridas al sustrato, que impiden con mayor eficacia el paso de iones agresivos hacia el sustrato y dan como resultado un sistema más resistente a la corrosión.

En definitiva, estos resultados corroboran el comportamiento observado tanto en las pruebas electroquímicas como en SEM y XRD, que atribuyen a la película de conversión obtenida en la solución fosfatante 1:5 un mejor comportamiento frente a la corrosión en medio clorurado, mostrando una mayor cobertura, homogeneidad, espesor y adherencia.

\section{CONCLUSIONES}

Los resultados indican que el tratamiento de fosfatado obtenido en la solución 1:5 genera una película de conversión muy delgada, con espesores de 1-2 $\mu \mathrm{m}$, principalmente constituida por fosfato de hierro secundario y pequeñas trazas de magnetita. Por otro lado, el fosfatado favorece un importante incremento de la rugosidad y, por tanto, de la microporosidad, modificando las características electroquímicas y de adherencia con respecto al acero desnudo.

Las muestras de acero fosfatadas revelan un incremento en la resistencia a la corrosión con respecto al acero desnudo. Los resultados en inmersión continua muestran que la aplicación de un recubrimiento orgánico sobre el sustrato previamente fosfatado en la dilución 1:5 (10 $\mathrm{min}$ ) aumenta la resistencia a la corrosión en, al menos, un orden de magnitud con respecto al sustrato no fosfatado e incrementa el tiempo de vida del sistema recubrimiento-sustrato. Este sistema presenta también los valores más altos de adherencia, aproximadamente 10 veces superior a la obtenida para el acero desnudo.

En definitiva, el fosfatado como TCQ muestra seguir siendo una alternativa viable y económica, libre de iones Cr (VI), que genera películas altamente adherentes al sustrato y resistentes a la corrosión, que podría ser utilizada como punto de partida para su posible aplicación en otros campos.

\section{Agradecimientos}

Los autores agradecen el apoyo económico del proyecto MAT2008-06719-C03-01. E. Onofre agradece también la ayuda recibida de la UNAM y el CSIC a través del programa científico de cooperación UNAM-CSIC, "Simon Bolivar" postdoctoral 2009. 2010, DGAPA-UNAM. M. Carboneras agradece la concesión de un contrato JAE-Doc otorgada por el CSIC.

\section{REFERENCIAS}

[1] G. Zhang, J. Huang, K. Yang y B.C. Zhang, Acta. Metall. Sin. 43 (2007) 1.186-1.192.

[2] B.Y. Jhonson, J. Edington y J. O'Keefe, Mater. Sci. Eng. 361 (2003) 225-231.

[3] F. William, G. O'keefe, J. Mattew y Z. Haifen, Surf. Coat. Technol., 155 (2002) 127-136.

[4] B. Chico, J.C. Galván, D. de la Fuente y M. Morcillo, Prog. Org. Coat. 60 (2007) 4553.

[5] A. Decroly y J. P. Petitjean, Surf. Coat. Technol. 194, (2005) 1-9.

[6] D. Chidambaram, C. Clayton y R. Halada, Electrochim. Acta 51 (15) (2006) 2.862-2.871.

[7] B.R.W. Hinton, J. Alloys. Comp. 180 (1992) $15-25$

[8] M.G.S. Ferreira, R.G. Duarte, M.F. Montemor y M.P. Simoes, Electrochim. Acta. 49 (2004) 2.927-2.935.

[9] B.R.W. Hinton y L. Wilson, Corros. Sci. 29 (1989) 967-975.

[10] C. Wang, F. Jiang y F. Wang, Corros. Sci. 46 (2004) 75-89.

[11] L. Jacobi, Macchia O Ind. Mecánica, 17, (1935) 137-142.

[12] R. R. Tanner, Monthly, Rev. Am. Electroplaters Soc. (1934) 21-29.

[13] M. Thornton-Murray, M. Eng. 85 (1908) 870882.

[14] T.W. Coslett, British Patent 8, 667, (1906).

[15] T.W. Coslett, British Patent 15, 628, (1908).

[16] T.W. Coslett, British Patent 22, 743, (1909).

[17] T.W. Coslett, British Patent 28, 131, (1909).

[18] L.E. Eckelmann, Chem. Metall. Eng. 21 (1919) 787-790.

[19] W.H. Allen, U.S. Patent 1, 206, 075, (1916).

[20] W.H. Allen, U.S. Patent 1, 311, 726, (1914). 
[21] V.M. Darsey y R.R. Tanner, U.S. Patent 1, 887, 967, (1932).

[22] X. Llovensá, R.M. Raurell y S.A. Hodges, Paint Coat. Ind. Mag. (2010) 34-37.

[23] R. Naderi y M. M. Attar, J. Appl. Electrochem. (2009) 2.353-2.358.

[24] R. Naderi y M. M. Attar, Prog. Org. Coat. (2009) 314-320.

[25] F.J.J. Kellner, K. Schütze, C. Kreutz y S. Virtanen, Surf. Interface Anal. 41 (2009) 911-917.

[26] Y. Hamlaoui, L. Tifouti y F. Pedraza, Corros. Sci. 51 (2009) 2.455-2.462.

[27] L. Fedrizzi, F. Deflorian, S. Rossi, L. Fambri y P.L. Bonora, Prog. Org. Coat. 42 (2001) 65-74.

[28] T.S.N. Sankara, Rev. Adv. Mater. Sci. 9 (2005) 130-177.

[29] R. Moore y B. Dunham, Met. Finish. 106 (2008) 46-55.

[30] C.G. Munger, Corrosion prevention by protective coatings, Ed. NACE, First edition, Houston, USA, 1984, pp. 87-96.

[31] R.M. Burns y W.W. Bradley, Protective Coatings for Metals, Ed. Reinhold Publishing Co., New York, USA, 1964, pp. 236-380.

[32] N.R. Roobol, Industrial painting principles and practices, Ed. Hanser Gardner Publications,
Second edition, Munich, Germany, 1997, pp. 346-420.

[33] N. Bretherton, G. E. Thompson y S. Turgoose, Corros. Sci. 35 (1993) 1.297-1.303.

[34] E. Onofre-Bustamante, M.A. DomínguezCrespo, J. Genescá-Llongueras, F.J. RodríguezGomez, Surf. Coat. Technol., (2007) 4.6664.676.

[35] D. B. Freeman, Phosphating and Metal Pretreatment - A Guide to Modern Processes and Practice, Ed. Industrial Press, First edition, New York, USA, 1986, pp. 123-180.

[36] W. Rausch, The Phosphating of Metals, Ed. Finishing Publications, London, England, 1990, pp. 96-123.

[37] C. G. Munger, Corrosion prevention by protective coatings, Ed. Nace, First edition Houston, USA, 1984, pp. 184-256.

[38] V.F. Cunha, G.F. Andrade, C.R. Araujo y T. Matencio, Appl. Surf. Sci. 253 (2006) 2.8752.884 .

[39] J. Genescá, Técnicas electroquímicas para el control y estudio de la corrosión, Ed. Universidad Nacional Autónoma de México, DF, México, 2002, pp. 45-70. 